\title{
Community treatment with azithromycin for trachoma is not associated with antibiotic resistance in Streptococcus pneumoniae at 1 year
}

\author{
B D Gaynor, K A Holbrook, J P Whitcher, S O Holm, H C Jha, J S P Chaudhary, \\ R C Bhatta, T Lietman
}

Br J Ophthalmol 2003;87:147-148

\begin{abstract}
Aims: To determine if macrolide resistant Streptococcus pneumoniae will be a major concern in areas that receive annual mass azithromycin distributions for trachoma.

Methods: A cross sectional survey was conducted of nasopharyngeal $S$ pneumoniae isolates for susceptibility to azithromycin 1 year after administering a single dose of azithromycin to treat trachoma in a village in Nepal.

Results: S pneumoniae was isolated from $50(86 \%)$ of 57 nasopharyngeal cultures and no resistance to azithromycin was detected.

Conclusion: The authors were unable to demonstrate that mass azithromycin therapy for trachoma produced macrolide resistant $S$ pneumoniae that persists until the next scheduled annual treatment.
\end{abstract}

$M$ ass, community-wide distributions of azithromycin have been shown to be effective in reducing the prevalence of ocular chlamydial infection in a population. ${ }^{1-6}$ Given the World Health Organization's (WHO) initiation of a worldwide programme using azithromycin to eradicate trachoma by the year $2020,{ }^{7}$ the recommendation for mass treatment, ${ }^{1}$ and the likely requirement for repeated treatments, ${ }^{8}$ additional consequences of this systemic treatment should be explored.

Potential disadvantages of systemic treatment include allergic or other reactions and the selection and propagation of macrolide resistant bacteria in treated populations. Determining macrolide resistance in Chlamydia trachomatis is not standardised and rarely pursued, although there is well documented resistance to both azithromycin and erythromycin in Streptococcus pneumoniae. ${ }^{9-11}$ After a single dose of azithromycin for trachoma, pneumococcal resistance has appeared in conjunctival isolates $^{12}$; however, it is unclear that their presence alone presents clinical concerns. In a longitudinal study, a low level baseline prevalence of macrolide resistant pneumococcus in a community increased after a single dose of azithromycin for trachoma. ${ }^{13}$

Given that annual treatment may be needed in trachoma endemic areas, ${ }^{8}$ timely assessments of nasopharyngeal $S$ pneumoniae isolates would provide insight into the threat of burgeoning resistance. If no resistance exists 1 year after treatment, then perhaps annual azithromycin treatment will not have a significant impact on the prevalence of azithromycin resistant $S$ pneumoniae in a population. In this study, the prevalence of macrolide resistant $S$ pneumoniae was assessed 1 year after treatment with azithromycin, immediately before retreatment.

\section{MATERIALS AND METHODS}

In a rural village in western Nepal, all children age $1-10$ years were examined for the presence of trachoma on 1 December 1998 (visit 1). Clinical activity was assessed during examination, based on the WHO simplified trachoma grading scale, ${ }^{14}$ and utilising a $2.5 \times$ loupe by trained personnel. All children with clinically active trachoma and all household members of these children were treated with a single dose $(20 \mathrm{mg} / \mathrm{kg}$ in children; $1 \mathrm{~g}$ in adults) of oral azithromycin (Zithromax, Pfizer, New York, NY, USA).

Approximately 1 year later, on 15 November 1999 (visit 2), all village children were re-examined for the presence of trachoma. In addition, nasopharyngeal specimens were collected, using a calcium alginate swab, from a random cross section of children aged $1-10$ years. Randomisation was achieved by assigning each child a pseudorandom number between 0 and 1 (Microsoft Excel "rand" function), and selecting all those with a value $<0.3$.

Nasopharyngeal specimens were immediately placed on selective streptococcus agar (blood agar with neomycin and polymixin B; Hardy Diagnostics, Santa Maria, CA, USA) and processed using standard microbiological techniques. Isolates were confirmed $S$ pneumoniae by growth on streptococcus selective media, $\alpha$ haemolysis, susceptibility to optochin, and bile salt solubility. MIC values for azithromycin and trimethoprim/sulfamethoxazole were determined using Etest (AB Biodisk, Piscataway, NJ, USA). Control isolates confirmed the reliability of antibiotic susceptibility testing.

\section{RESULTS AND DISCUSSION}

At visit 1, a total of 243 children were examined and 94 (39\%) were found to have clinically active trachoma. On visit 2,21 of $175(12 \%)$ of the village children were found to have clinically active trachoma. Nasopharyngeal cultures were performed on 57 (median age 6 years, range 1-10) of the 175 children. Fifty of the 57 nasopharyngeal swabs were positive for $S$ pneumoniae (prevalence $86 \% ; 95 \%$ CI, 76\% to 95\%). None of these isolates ( $0 \%$; $95 \%$ CI, $0 \%$ to $15 \%$ ) were resistant ( $\mathrm{MIC} \geqslant 2 \mu \mathrm{g} / \mathrm{ml}$ ) to azithromycin. Four of the 50 isolates ( $8 \%$; $95 \%$ CI, $2 \%$ to $20 \%$ ) were resistant (MIC $\geqslant 4 \mu \mathrm{g} / \mathrm{ml}$ ) to trimethoprim/ sulfamethoxazole.

There is a high prevalence of nasopharyngeal colonisation with $S$ pneumoniae in the children of this rural Nepali village $(86 \%)$. The $8 \%$ resistance to trimethoprim/sulfamethoxazole in S pneumoniae is not surprising as this antibiotic is one of the most widely available antibiotics in the area. Macrolide resistant $S$ pneumoniae were not present in this sample of children 1 year after community-wide treatment with azithromycin. However, because of the low expected frequencies of macrolide resistance and the small sample size, these data lack power-even if as many as $15 \%$ of infections (or colonisations) with $S$ pneumoniae in this village were resistant to macrolides, 
there would still be a 5\% chance of having recorded no resistance in our sample.

There was no control village that had not received azithromycin. However, the government sub-health posts in this area prescribe amoxacillin and trimethoprim/sulfamethaxozole, but not macrolides. Thus, the chance of de novo resistance in villages that did not receive azithromycin may be quite low.

In the study by Leach et al, azithromycin resistance was found in $1.9 \%$ of the pneumococcal isolates at baseline, $55 \%$ at 2-3 weeks, $35 \%$ at 2 months, and $6 \%$ at 6 months after azithromycin treatment. ${ }^{13}$ Resistant serotypes that were found at 2-3 weeks, were less prevalent at 2 months and 6 months, perhaps because they were replaced by sensitive wild types. It is tempting to speculate that prevalence of these resistant strains might have returned completely to the baseline level at l year, which would be consistent with the current study's data.

Infectious diseases are transmissible, so if an individual in a village acquires a drug resistant strain, others in the same village are more likely to be infected with the same strain; samples taken from different villages will not correlate as well as samples taken from the same village. Thus, the above confidence intervals account for the sampling error of the prevalence within that particular village only, and do not reflect what would be expected by assessing other (even identical) villages which had undergone similar treatments. More villages, rather than just more people from a single village, need to be monitored before the risk for unacceptable levels of drug resistance can be discounted with confidence.

This study did not definitively prove the absence of resistance at 1 year. Nevertheless, the data are encouraging. Effective therapy for trachoma has not produced an unanticipated amount of macrolide resistant $S$ pneumoniae in these children. If little or no resistance is found 1 year after treatment, the chance of establishing a permanent population of resistant bacteria is probably low. However the effect of repeat treatment is unknown, and assessment after multiple treatments is necessary. Resistance in villages where the entire population is treated with macrolides should be assessed. Only about $50 \%$ of the children were treated in both this study and in Leach's study. ${ }^{2}{ }^{13}$ Finally, even if a significant increase in the prevalence of resistant pneumococcus had been found 1 year after mass antibiotic administration, the benefit of trachoma eradication would probably outweigh the potential risks associated with the presence of resistant pneumococcus in these populations. Resistant organisms tend to be less fit than the wild type. Their presence is transitory and probably would reverse when treatment programmes conclude. ${ }^{15}$ Larger longitudinal studies of multiple villages and multiple treatments are currently under way to address the limitations of this pilot study.

\section{ACKNOWLEDGEMENTS}

This project was carried out through the generous support of the Alta Foundation, Geta Eye Hospital, Helen Keller International (HKI), Nepal Netra Jyoti Sangh (NNJS; National Society for Comprehensive Eye Care), the National Institute of Allergy and Infectious Diseases (Grant K08 AI 01441), the South Asia Research Fund, Research to Prevent Blindness, and the FI Proctor Foundation. Pfizer Inc generously donated the azithromycin for the trachoma programme in
Kailali District, Nepal. We would like especially to thank the following people for their invaluable help with the project: members of Geta Eye Hospital including RR Bhatta, Jamuna BK, RB Chaudhary, GB Chaudhary, BR Chaudhary, BS Dhami, Batti Gurung, Manju Gurung, LR Panta, KS Khuna, and TB Deupa; the staff from both HKI and ECARDS, in particular Bal Bahadur and Ganga Rana, Diwash Rijal of NNJS, HS Bista of Norwegian Church Aid, and Stephanie Costanza of the Proctor Foundation.

\section{Authors' affiliations}

B D Gaynor, J P Whitcher, T Lietman, The Francis I Proctor Foundation and the Department of Ophthalmology, University of California, San Francisco, San Francisco, CA, USA

K A Holbrook, Community Health Epidemiology and Disease Control, San Francisco Department of Public Health, San Francisco, CA, USA SO Holm, The Francis I Proctor Foundation

H C Jha, J S P Chaudhary, R C Bhatta, Geta Eye Hospital, Geta, Nepal

Correspondence to: Bruce D Gaynor, MD, The Francis I Proctor Foundation and the Department of Ophthalmology, UCSF Medical Center, San Francisco, CA 94143-0944, USA; bgaynor@itsa.ucsf.edu

Accepted for publication 19 March 2002

\section{REFERENCES}

1 Schachter J, West SK, Mabey D, et al. Azithromycin in control of trachoma. Lancet 1999;354:630-5.

2 Laming AC, Currie BJ, Difrancesco $M$, et al. A targeted, single-dose azithromycin strategy for trachoma. Med J Aust 2000;172:163-6.

3 Bailey RL, Arullendran P, Whittle HC, et al. Randomised controlled trial of single-dose azithromycin in treatment of trachoma. Lancet 1993;342:453-6.

4 Dawson CR, Schachter J, Sallam S, et al. A comparison of oral azithromycin with topical oxytetracycline/polymyxin for the treatment of trachoma in children. Clin Infect Dis 1997;24:363-8.

5 Tabbara KF, Abu-el-Asrar A, al-Omar O, et al. Single-dose azithromycin in the treatment of trachoma. A randomized, controlled study. Ophthalmology 1996;103:842-6.

6 Osaki Holm S, Jha HC, Bhatta RC, et al. A comparison of two azithromycin distribution strategies for trachoma. Bull World Health Organ 2001 (in press).

7 World Health Organization. Report of the first meeting of the WHO alliance for the global elimination of trachoma. Geneva: WHO, 1997

8 Lietman T, Porco T, Dawson C, et al. Global elimination of trachoma: how frequently should we administer mass chemotherapy? Nat Med 1999:5:572-6.

9 Oster P, Zanchi A, Cresti S, et al. Patterns of macrolide resistance determinants among community-acquired Streptococcus pneumoniae isolates over a 5 -year period of decreased macrolide susceptibility rates. Antimicrob.Agents Chemother 1999;43:2510-2.

10 Doern GV, Pfaller MA, Kugler K, et al. Prevalence of antimicrobial resistance among respiratory tract isolates of Streptococcus pneumoniae in North America: 1997 results from the SENTRY antimicrobial surveillance program. Clin Infect Dis 1998;27:764-70.

11 Thornsberry C, Jones ME, Hickey ML, et al. Resistance surveillance of Streptococcus pneumoniae, Haemophilus influenzae and Moraxella catarrhalis isolated in the United States, 1997-1998. J Antimicrob Chemother 1999;44:749-59.

12 Chern KC, Shrestha SK, Cevallos V, et al. Alterations in the conjunctival bacterial flora following a single dose of azithromycin in a trachoma endemic area. Br J Ophthalmol 1999:83:1332-5.

13 Leach AJ, Shelby-James TM, Mayo M, et al. A prospective study of the impact of community-based azithromycin treatment of trachoma on carriage and resistance of Streptococcus pneumoniae. Clin Infect Dis 1997;24:356-62.

14 Thylefors B, Dawson CR, Jones BR, et al. A simple system for the assessment of trachoma and its complications. Bull World Health Organ 1987; 65:477-83

15 Seppala H, Klaukka T, Vuopio-Varkila J, et al. The effect of changes in the consumption of macrolide antibiotics on erythromycin resistance in group A streptococci in Finland. Finnish Study Group for Antimicrobial Resistance. N Engl J Med 1997;337:441-6. 